\title{
Reconstructing past mantle flow in the South Atlantic
}

\author{
LORENZO COLLI ${ }^{1}$
}

${ }^{1}$ Department of Earth and Atmospheric Sciences, University of Houston, Houston, TX, USA. E-mail: 1colli@uh.edu

Recent studies have shown that the South Atlantic has experienced a complex evolution since the breakup of Gondwana that does not fit what is predicted by simple thermal relaxation following continental rifting. The geologic record clearly shows post-rift phases of renewed uplift, diffuse magmatism and rapid changes in spreading rates, which cannot be easily explained by lithospheric-scale processes. Instead, these observations suggest an important and active contribution from global mantle convection and viscous flow in the asthenosphere, as illustrated by simple scaling arguments.

The interplay between mantle dynamics and surface geology can in principle be quantified more explicitly and more precisely via global geodynamic models of mantle convection. But in practice the usefulness of such models is currently limited by uncertain model parameters and by the lack of a good initial condition in the past. This second problem can be overcome by solving a geodynamic inverse problem aimed at finding the optimal initial condition which evolves into an estimated presentday state of the mantle. The history of mantle flow so obtained is unique and can be used to test uncertain model parameters and geophysical hypotheses.

In this talk I will first review how simple analytical solutions help us explain various geologic observations in the South Atlantic. I will then move on to discuss the scientific potential of explicit reconstructions of past mantle flow obtained by solving the geodynamic inverse problem via the adjoint method and their application to the South Atlantic. 